ISSN 1112-9867

Available online at

http://www.jfas.info

\title{
SIG-CLIMALG: THE GEOGRAPHICAL DIMENSION OF CLIMATE DATA IN ALGERIA
}

\author{
T. Bennour ${ }^{1}$, M. S. Guettouche ${ }^{1}$, E. Matinez Ibrra ${ }^{2, *}$ \\ ${ }^{1}$ Laboratoire de Géomorphologie et géorisques, USTHB, Algerie \\ ${ }^{2}$ Département de l'analyse géographique régionale et physique, Université de Granada, \\ Espagne
}

Received: 20 October 2016 / Accepted: 15 July 2017 / Published online: 01 September 2017

\begin{abstract}
In Algeria, implementation, monitoring and exploitation of climate data, development and dissemination of periodic publications and climate assistance to users, are tasks of the National Climatological Centre (NCC). Moreover, the effective management of this large volume of data requires the use of new technologies such as Geographic Information Systems (GIS). In this context, the aim of the present work is to develop an IT solution on the Web, based on the use of Geographic Information Systems. The approach consists, first, to design databases to store various data used (climatic and topographic) considering the functional needs such as the dissemination of data in several modes (read, download) and the possibility direct data access from remote locations. Then the choice of technology and tools to implement a mapping solution that will allow, in addition to the functionality of storage and distribution of different types of data, analysis of climate observations by using a web service whitin the WPS (Web Processing Service) standard.
\end{abstract}

Keywords: GIS, SIG-CLIMALG, climate data, WPS

Author Correspondence, e-mail: tbennour@usthb.dz doi: http://dx.doi.org/10.4314/jfas.v9i3.1 


\section{INTRODUCTION}

L'office National de la Météorologie (ONM) est un établissement public à caractère industriel et commercial, créé en 1975 sous la tutelle du ministère des transports. Il a pour mission la mise en œuvre de la politique nationale en matière de météorologie et de contribuer à la sécurité et au développement du secteur socio-économique. L'ONM est composé de quatre départements techniques dont le Centre Climatologique National (CCN) qui a pour vocation principale l'acquisition auprès de ses directions régionales et d'autres sources, le contrôle, le traitement, l'exploitation et la publication des données météorologiques nationales et internationales ainsi que l'assistance aux usagers (ONM, 2015). A ce titre, et afin de gérer efficacement ces données, le $\mathrm{CCN}$ a mis en place un système reliant ses directions régionales localisées dans des lieux distants sur le territoire national. Ce système comprend des moyens de communication pour la collecte, l'échange et la diffusion des données en temps réel (ONM, 2015).

D'autre part, l'avancée technologique a donné un nouvel essor aux Systèmes d'Information (SI). Un SI est un ensemble organisé de ressources (matériel, logiciel, personnel, données, procédures...) permettant d'acquérir, de traiter, de stocker des informations dans et entre des organisations [6]. S'agissant des Systèmes d'Information Géographiques (SIG), beaucoup d'auteurs les considérèrent comme des cas particuliers des SI en général [17], et d'autres les considèrent comme une des partie du SI, qui se compose de divers applications et ils peuvent prendre en considération la dimension géographique [7].

De plus, ces dernières années ont été marquées par l'essor d'une nouvelle forme de SIG reposant sur les techniques du Web 2.0, ce qui a permet aux utilisateurs, non seulement de consulter des représentations géographiques de l'information publier sur le web, mais aussi de participer et collaborer au contenu en créant toute sorte d'information géographique [5], ces outils sont désormais appelés Systèmes d'Information Géographique sur le Web (SIGWeb).

Dans ce contexte, le présent travail fait partie d'un projet d'envergure qui consiste à développer un système fédérateur englobant une multitude de composants du système actuel, afin d'assurer une interopérabilité entre les systèmes installés au niveau de chaque structure régionale et le système central. Notre contribution consiste à proposer une solution 
informatique reliant les structures régionales avec la structure centrale, le choix s'est porté sur les SIGWeb car, ils incluent en plus aux fonctionnalités classiques de visualisation des cartes, des fonctions de requêtes attributaires et spatiales ou même des géotraitements [8]. Notre SIGWeb permettra l'analyse cartographique et statistique des observations climatiques ainsi que l'archivage, l'échange et la diffusion des différents types de données (surfacique, d'altitude, satellitaire, radar et autres).

L'objectif de ce travail est de répondre à un besoin exprimé par le Centre Climatologique National, afin de remédier aux problèmes liés à l'échange d'un volume important de données hétérogènes entre la structure centrale et ses structures régionales localisées dans des sites éloignés sur un territoire caractérisé par un relief très varié d'une région à une autre.

\section{MÉTHODOLOGIE}

Notre contribution consiste, au premier lieu, de proposer une méthodologie permettant de mettre en place une solution informatique pour stocker, partager, publier et exécuter des analyses spatiales sur des données climatiques. Ensuite, de collecter toutes les informations nécessaires auprès des institutions productrices de données, et de les organiser au sein des bases de données. Enfin, d'implémenter la solution avec des outils Open Source et de développer les fonctionnalités d'analyses spatiales nécessaires.

Pour faire face aux exigences fonctionnelles de l'utilisateur, nous avons établi une méthodologie simple avec comme trame principale de fournir le maximum possible d'informations relatives au climat au profit des utilisateurs distants, via un outil informatique convivial et efficaces utilisable, même par un non informaticien. Le choix s'est arrêté sur les SIGWeb en raison de leurs capacités de rassembler une panoplie de données géographiques et sémantiques ainsi que, pour ses interfaces cartographiques facilement manipulables par un non géomaticien.

Les SIGWeb disposent les mêmes fonctionnalités que les SIG bureautiques, souvent dénommées les cinq (5) A : Abstraction, Acquisition, Archivage, Analyse et Affichage [16]. Pour aboutir à un puissant Système d'Information Géographique répondant aux besoins de l'utilisateur, ces fonctionnalités doivent être mises en œuvre : 
1. Abstraction : Une base de données est l'ensemble de données modélisant les objets du monde réel et liés par des relations, elles sont interrogeables selon n'importe quel critère [3]. Ainsi, l'abstraction est la modélisation de problème sous forme d'une base de données afin de le rendre compréhensible par les utilisateurs. Dans ce cas, nous avons utilisé le langage de modélisation UML (Unified Modeling Language).

2. Acquisition: Cette étape est dédiée à la collecte des différentes informations relatives au climat qui sont nécessaires au fonctionnement du système. Il est à signaler que, dans un projet SIG, les données représentent 60 à $80 \%$ de son coût, il importe donc de bien spécifier les besoins afin d'optimiser les investissements [16]. Nos données sont acquises auprès de plusieurs institutions et sous différentes formes qui seront présentées ultérieurement.

3. Archivage : L'archivage vise le stockage de manière rationnelle les informations acquises afin de les exploiter facilement. Cette fonctionnalité est assurée par un ensemble de logiciels systèmes appelé les Systèmes de Gestion de Bases de Données (SGBD) qui permettent aussi aux utilisateurs d'insérer, de modifier et de rechercher efficacement des données spécifiques dans une grande masse d'informations partagée par de multiples utilisateurs [3]. Ce volet sera traité en détail dans la section « organisation des données ».

4. Analyse : C'est le cœur d'un SIG, elle se fait par les outils d'analyse spatiale pour interroger la base de données afin de répondre aux requêtes des utilisateurs. Aussi, ces outils sont plus puissants quand les relations topologiques sont stockées dans la structure de données [18]. Certains de ces outils sont disponibles en standard avec les logiciels de la plateforme SIGWeb et leur utilisation requière un simple paramétrage pour répondre, généralement, aux requêtes non thématiques, néanmoins, la majorité des outils d'analyse spatiale thématique (climatique dans notre cas) nécessitent un développement spécifique. Ces deux cas seront traités dans notre application.

5. Affichage: C'est la partie visible pour l'utilisateur où le système lui restitue des informations à partir de la base de données, sous forme de cartes, des diagrammes ou des rapports, c'est la dernière étape de tout projet SIG [9]. 


\section{L'ARCHITECTURE DU SYSTÈME}

Une architecture du système est une structure composée de modules actifs, d'un mécanisme d'interaction entre ces modules et d'un ensemble de règles qui gèrent cette interaction [1]. Le choix de l'architecture doit se faire d'une manière efficace pour satisfaire les besoins fonctionnels exprimés par le client (les fonctionnalités du système), et non fonctionnels liés aux contraintes d'implémentation du système (optimisation des ressources,...etc).

Dans notre travail, nous avons opté pour une architecture client/serveur trois tiers. Le principe de cette architecture consiste à séparer la réalisation des trois groupes de fonctions nécessaires pour tout système d'information, à savoir : le stockage des données, la logique applicative et la présentation afin d'assurer une évolutivité du système. Cette séparation peut être physique, cela signifie qu'il est possible de déployer chaque fonction sur un serveur indépendant. Ainsi, leur déploiement sur la même machine ne présente pas d'inconvénient pour les petits systèmes.

Les trois éléments permettant la réalisation de cette architecture sont :

- Le système de gestion de base de données relationnel : dédié au stockage des données de façon structurée ;

- Le serveur applicatif pour la logique applicative : cette partie utilise les données pour les présenter de façon exploitable par l'utilisateur. Dans notre cas, il s'agit de deux serveurs web et cartographique, ce dernier prenait généralement la forme d'une couche logicielle (souvent composée de différentes applications) installée sur la machine serveur, qui reçoit les requêtes de type SIG et complète les réponses du logiciel serveur par des éléments cartographiques [19];

- Le navigateur web pour la partie de présentation : elle a une importance primordiale, car c'est la partie la plus visible pour l'utilisateur.

Pour l'analyse et la conception du système nous avons utilisé le langage de modélisation UML. Il propose, dans sa version 2, treize diagrammes qui peuvent être utilisés dans la description d'un système, ces diagrammes sont regroupés dans deux grands ensembles, structurels et de comportement [4]. Le concepteur a la possibilité de choisir les diagrammes à réaliser afin de représenter les besoins du système à développer. La figure 1 représente le 
diagramme de paquetage (un des diagrammes structurels) qui permet de décomposer le système en parties plus facilement observables, appelés «paquetage». Dans notre cas, le système peut être divisé en quatre paquetages (composantes) : le système de gestion de base de données, le serveur cartographique, le serveur web et le client web.

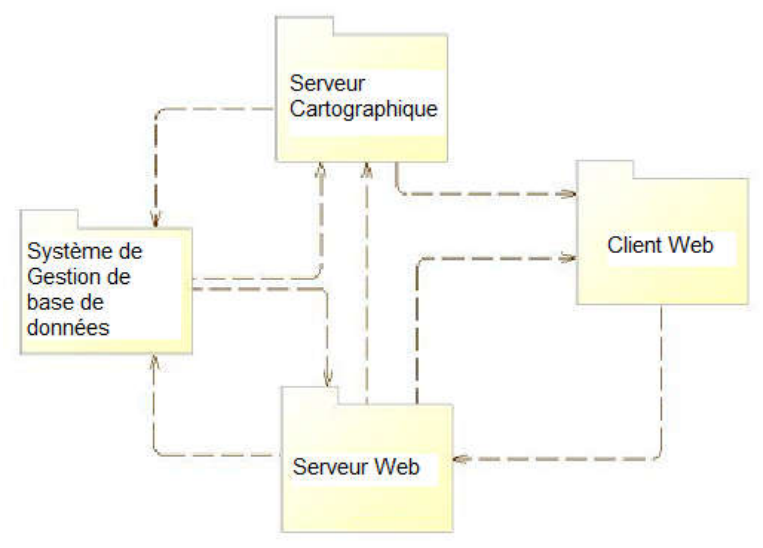

Fig.1. Architecture du système (diagramme UML de paquetages)

\section{L'IMPLÉMENTATION DE LA SOLUTION}

\subsection{La structuration des données utilisées}

En plus à la capacité des SIG de gérer simultanément le spatial et le sémantique et leurs liens au sein d'une base de données géographiques, ces outils permettent aussi des traitements statistiques [15]. Dans notre cas, l'utilisation d'un SIG s'impose d'elle-même car nos données sont hétérogènes et issues de multiples sources et de natures différentes, leur structuration dans des bases de données permettra de répondre efficacement à une grande variété de questions.

Les données à intégrer dans un SIG se décomposent en deux catégories: les données thématiques et celles de références [14]. La première catégorie est la partie principale (opérationnelle) sur laquelle le système s'appui pour répondre aux requêtes des utilisateurs, telles que : quelle est la plus haute température enregistrée dans la ville de Laghouat durant les dix dernières années? Ou encore, quelles sont les régions de l'Algérie les plus exposées aux tempêtes de sable ? Ces données thématiques correspondent aux données climatiques de l'Algérie, et sont sous forme graphique (carte, graphe, etc.), ou sous formes numérique (tableaux). 
Les données de références servent comme un font cartographiques pour le positionnement des données thématiques, elles sont représentées par des cartes topographiques vectorielles et des images satellitaires. La figure 2 montre les types des données utilisées ainsi que leurs sources.

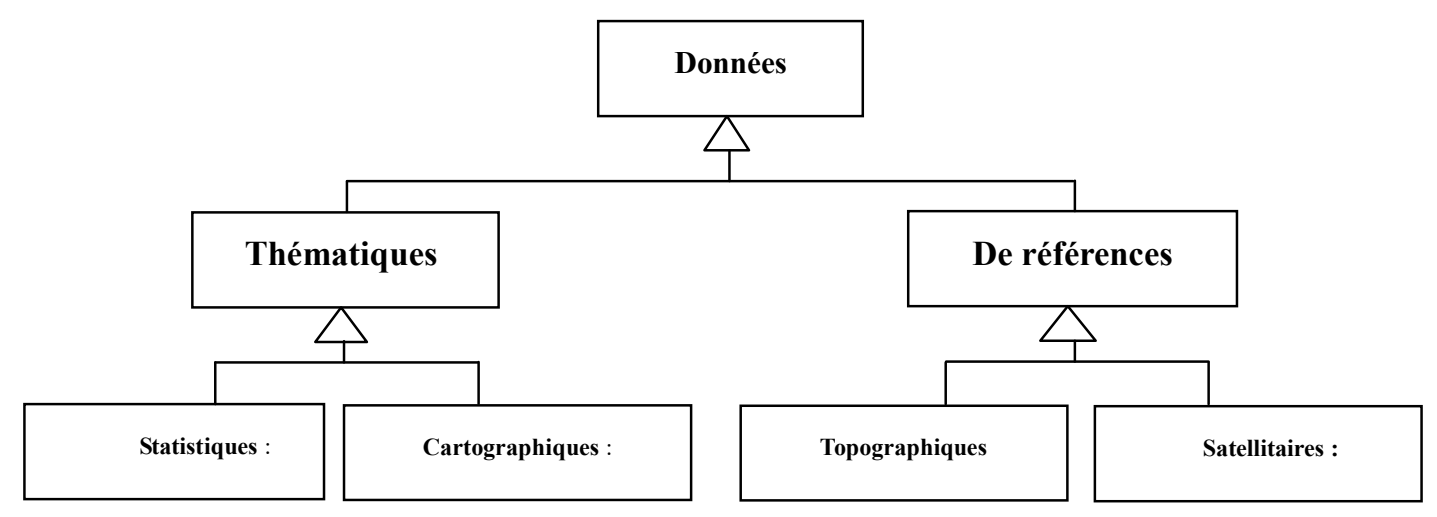

Fig.2. Les types des données utilisées

\subsubsection{Les données thématiques}

\section{A. Les données statistiques}

Il s'agit ici des mesures statistiques sur les données atmosphériques d'une période de vingt et un (21) ans (1987-2007) des quatre stations météorologiques professionnelles de 1'ONM suivantes : Ghazaouet, Alger, Annaba et Laghouat (tableau 1, Figure 3). Nous nous sommes limités uniquement à ces stations à cause de la disponibilité de leurs données.

Ces données ont été stockées dans la Base de Données Climatique Statistique (BDCS) sous forme tabulaire, en vue de leur utilisation par le système pour répondre aux requêtes des utilisateurs par des tableaux de calculs statistiques ou des graphes dans l'interface cartographique. En outre, ces données statistiques ont été exploitées en utilisant le logiciel QGIS, et ses méthodes d'interpolation spatiale qui permettent d'estimer les valeurs des points inconnus par rapport au données des quatre stations météorologiques sélectionnées, et ce pour créer des cartes statiques de température, de pluviométrie, de brouillard et de tempêtes de sable avant leur publication sous forme d'un service web de cartographie (WMS : Web Map Service).

Ci-dessous, une description des phénomènes et leurs données statistiques associées, qui sont 
pris en compte dans notre système :

- La température $\left({ }^{\circ} \mathbf{C}\right)$ : C'est un paramètre fondamental en météo, elle s'exprime en degré Celsius $\left({ }^{\circ} \mathrm{C}\right)$, elle décroit avec l'altitude d'environ 6 à $8{ }^{\circ} \mathrm{C}$ tous les $1000 \mathrm{~m}$ [11]. Nos données sont représentées dans un tableau qui contient les températures quotidiennes, enregistrées dans les régions voisines à la station sélectionnée, dans un intervalle d'une heure de temps. Ces données servent au calcul de la température moyenne annuelle, les températures minimale et maximale moyennes,...etc.

- La pluviométrie $(\mathrm{mm})$ : un tableau de statistiques représentant les hauteurs de précipitations en $\mathrm{mm}$ (millimètre). Le « $\mathrm{mm} »$ correspond en volume, à une hauteur d'eau de $1 \mathrm{~mm}$ sur une surface plane de $1 \mathrm{~m}^{2}$ (soit $1 \mathrm{~L}$ ). A partir de ce tableau, le système calcul la moyenne mensuelle de pluie, la moyenne annuelle de pluie...etc.

- L'humidité de l'aire (\%) : L'humidité est la quantité d'eau existée dans l'air sous forme de vapeur, incolore et inodore. Généralement, il s'agit de l'humidité relative (U) exprimée en \%, cette quantité de vapeur dépend de la température de l'air [10]. Dans notre cas, il s'agit d'un tableau représentant les mesures d'humidités enregistrées quotidiennement durant les vingt et un ans.

- La pression atmosphérique $(\mathrm{hPa})$ : C'est le poids de colonne d'air s'exerçant sur une surface déterminée, elle s'exprime en hectopscal $(\mathrm{hPa})$, et sa valeur décroit rapidement avec l'altitude [11]. Dans notre cas, il s'agit d'un tableau des mesures de pressions atmosphériques moyennes pour chaque station.

- La vitesse du vent $(\mathrm{m} / \mathrm{s})$ : c'est la vitesse du vent moyenne quotidienne en mettre/seconde $(\mathrm{m} / \mathrm{s})$ enregistrée par les stations sélectionnées. Quelques données manquantes ont été rattrapées par le calcul des moyennes arithmétiques de ses valeurs précédentes et suivantes.

- Les orages (jours) : Un orage est un phénomène atmosphérique se forme lorsque ce dernier est instable, il est caractérisé par une série d'éclairs et de coups de tonnerre. Il est accompagné par des phénomènes violents : précipitations intenses, rafales de vent,...etc (MeteoF, 2016). Les statistiques récupérées auprès de l'ONM, représentent 
le nombre moyen de jours d'orages enregistrés dans la région couverte par la station sélectionnée.

- Le brouillard (jours) : Ces données représentent le nombre moyen de jours de brouillard par mois durant les vingt et un ans considérées, et car ce phénomène est rare dans les régions sahariennes, ces données sont parvenues seulement des trois stations météorologiques côtières.

- Les tempêtes de sable (jours) : au contraire au phénomène précédent, les tempêtes de sables sont très souvent enregistrées dans les régions de sud et rares au nord, elles sont associées pleinement la dynamique éolienne et le transport des particules [12]. A cet effet, ces données concernent particulièrement la station de Laghouat.

Tableau1. Coordonnées des stations étudiées

\begin{tabular}{|c|c|c|c|}
\hline Stations & Altitude $(\mathbf{m})$ & Latitude (degré, min) & Longitude (degré, min) \\
\hline Ghazaouet & 83 & $35^{\circ} 06^{\prime} \mathrm{N}$ & $1^{\circ} 52^{\prime} \mathrm{W}$ \\
\hline Alger & 25 & $36^{\circ} 41^{\prime} \mathrm{N}$ & $3^{\circ} 13^{\prime} \mathrm{E}$ \\
\hline Annaba & 4 & $36^{\circ} 50^{\prime} \mathrm{N}$ & $7^{\circ} 49^{\prime} \mathrm{E}$ \\
\hline Laghouat & 762 & $33^{\circ} 46^{\prime} \mathrm{N}$ & $02^{\circ} 55^{\prime} \mathrm{E}$ \\
\hline
\end{tabular}

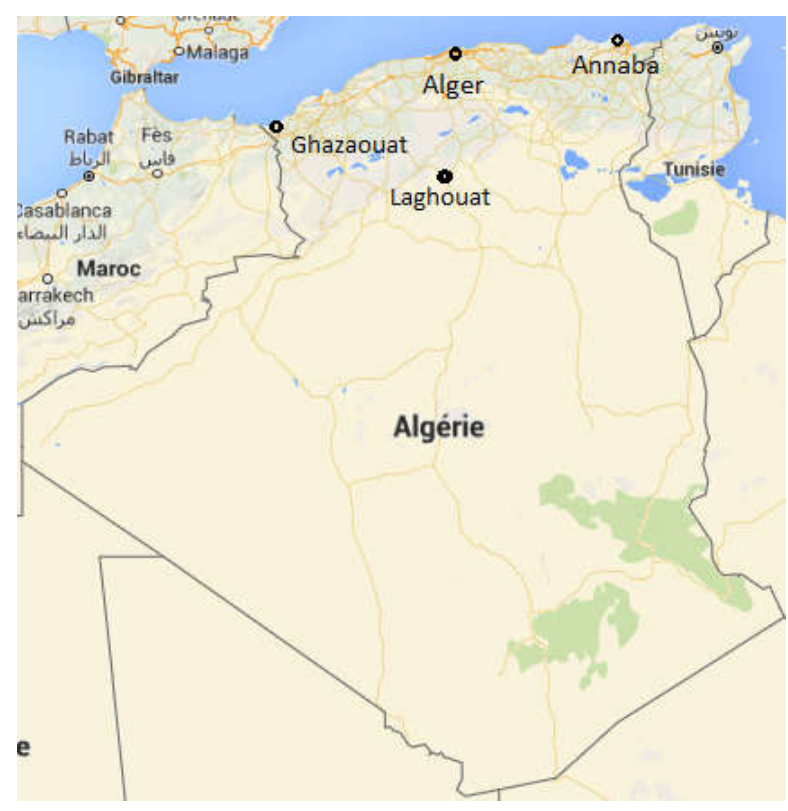

Fig.3. Localisation des stations météorologiques étudiées (source : google maps) 


\section{B. Les données cartographiques}

Ce type de données concerne principalement toutes les cartes numériques récupérées auprès des deux institutions Algériennes :

1. Les cartes produites par l'Agence Nationale des Ressources Hydrauliques (ANRH) :

- La carte pluviométrique du nord de l'Algérie : il s'agit d'une carte au 1:500 000 réalisée en 1993 par l'ANRH [24], elle représente les précipitations annuelles moyennes ramenées à une période de 60 ans (1921-1960 et 1968-1989) [13]. Elle est réalisée suivant une méthode proposée par J-P Laborde de l'université de Nice-Sophia Antipolis en France [22] ;

- Carte des évapotranspirations du Nord de l'Algérie : L'évapotranspiration est l'ensemble des phénomènes d'évaporation et de transpiration, elle désigne les pertes en eau par retour direct à l'atmosphère sous forme de vapeur d'eau [20]. Elle représente une part importante du cycle hydrologique car, environ 70 à $75 \%$ du total de la précipitation annuelle retourne à l'atmosphère par ce phénomènes (Guide, 2008). L'évapotranspiration est liée aux nombreux facteurs tels que : la température de l'air, la température de l'eau, l'humidité de l'air, le rayonnement solaire, le vent et la pression atmosphérique [20];

Cette carte au 1:500 000 a été réalisée par l'ANRH en 2002 [25], sur la base des données climatiques (température et humidité) de l'air, fournies par l'ONM, elles sont provenues de plus de 40 stations météorologiques réparties sur le territoire algérien. La durée d'observation de ces éléments météorologiques s'étale sur vingt et un ans (1988-2008) [2];

- La carte du réseau hydroclimatologique et de surveillance de la qualité des eaux : Cette carte au 1:500 000 est réalisée par le Département des Eaux Superficielles de l'ANRH en 2005 [26], elle contient des informations liées à l'implantation des stations pluviométriques, pluviographiques et climatologiques du nord de l'Algérie ;

- Carte des écoulements moyens annuels sur le nord de l'Algérie : Cette carte au 1:500 000 dressée par l'ANRH en 2005, estime les lames d'eaux moyennes annuelles écoulées en 
surface pour des bassins versants de superficie inférieure à $1500 \mathrm{~km}^{2}$. Elle est basée sur l'utilisation d'un modèle hydrologique calé sur les observations de 70 stations.

2. Cartes produites par le Centre de Développement des Énergies Renouvelables (CDER):

- La carte d'ensoleillement annuel en Algérie : Le territoire de l'Algérie est très étendu et englobe des régions climatiques très différentes, ce qui est valable aussi pour l'ensoleillement. La présente carte montre le nombre d'heures d'ensoleillement annuel qui peut aller jusqu'à 3550 heures par an au sud de l'Algérie. Elle a été réalisée par le CDER en 2010 (CDER, 2015).

- La carte de l'irradiation globale moyenne annuelle reçue sur une surface horizontale : Cette carte présente l'estimation des irradiations solaires incidentes sur plan horizontal par l'utilisation du modèle de Perrin de Brichambaut (CDRE, 2015).

Il est à signaler que ces cartes sont disponibles sur les sites web des deux institutions sous forme d'image statiques, ce qui signifie qu'aucune interrogation n'est possible sur ces cartes. Après leur géoréférencement et intégration dans la Base de Données Géographiques Raster (BDGR), créée pour héberger toutes les données de ce type, nous avons procédé à la vectorisation manuelle de ces cartes afin de rendre chaque objet de la carte manipulable de façon indépendante du reste du plan. En conséquence, chaque objet est devenu parfaitement autonome et constitue alors une entité identifiable à laquelle il est possible de rattacher des attributs, ce qui signifié que nos cartes sont transformées en cartes interactives. Ensuite, les couches produites ont été intégrées dans la Base de Données Géographiques Vectorielles (BDGV). Cependant, l'invisibilité de certaines cartes d'origine (avant vectorisation), notamment celles de l'ANRH, a constitué une contrainte majeure pour une bonne digitalisation et un bon renseignement des couches résultantes.

\subsubsection{Les données de références}

\section{A. Les données topographiques vectorielles}

Dans ce volet, nous avons utilisé les données numériques vectorielles à l'échelle 1:50 000 (Projection : UTM, Système géodésique : Nord Sahara 1959, Ellipsoïde : Clarke 1880) produites par l'Institut National de Cartographie et de Télédétection Algérien (INCT-Algérie). Ces cartes ont été réalisées sur la base des levés photogrammétriques issus des prises de vues 
aériennes au 1:50 000, suivi d'un complètement effectué sur le terrain (INCT, 2015), Ainsi que, les cartes produites par l'Agence Spatiale Algérienne (ASAL). Ces cartes se présentent sous forme de plus de 600 feuilles, toutefois, elles ne couvrent pas tout le territoire national. Ensuite, nous avons conçu et créé la Base de Données Topographiques (BDTopo50k) dont la structure des données est représentée par la figure 4, elle se compose de huit (08) thèmes principales, dont chaque thème a deux ou trois couches (Ponctuelle, linaire et surfacique). Par exemple, pour le thème «Com » qui indique «voies de communications » on trouve deux couches d'objets, une couche ponctuelle pour «les ponts» et une couche linaires pour «les routes et la voie ferrée».

\section{B. Les images satellitaires}

Des images satellitaires géoréférencées (Géotiff) d'une résolution de $10 \mathrm{~m}$ et prêtes à une intégration dans la BDGR, ont été acquises auprès de l'Agence Spatiale Algérienne (ASAL) selon nos besoins. Ce sont des images en provenance de satellite Alsat-2A/Algérie, en mode Multispectral à quatre bandes spectrales couvrant respectivement les fenêtres spectrales 0,45 -

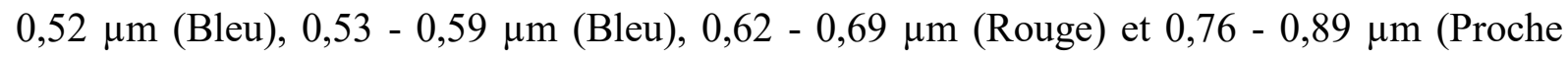
Infrarouge). Elles ont été utilisées pour les superposer sur les cartes de base afin de fournir aux utilisateurs une représentation holiste de la zone d'étude, ainsi que pour mettre à jour d'autres données géographiques de la BDTopo50k, notamment la couche «hydro» qui représente une couche fondamentale dans notre SIGWeb, ainsi que la couche «cons » pour saisir l'emplacement des moyens de communications des différents structures du CCN. 


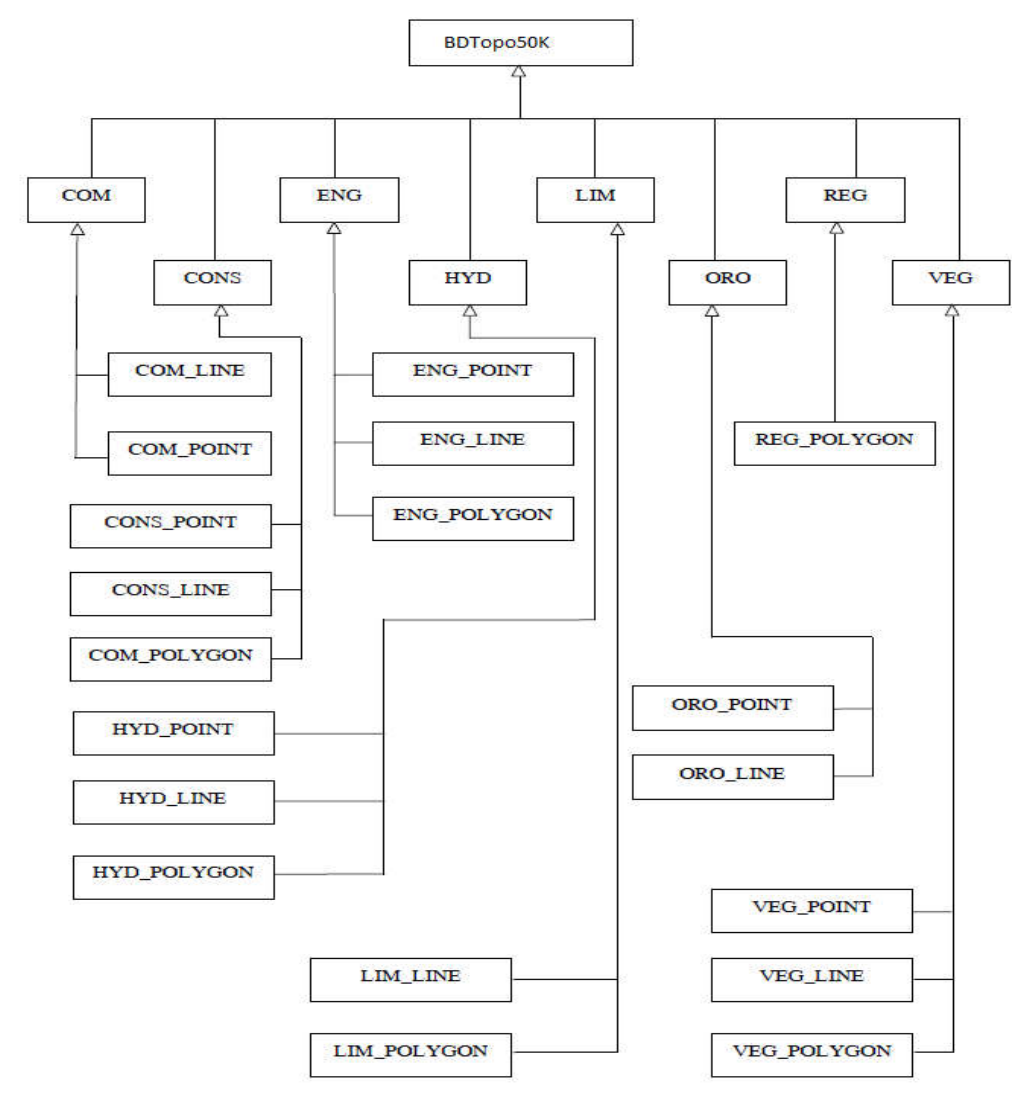

Com : voies de communications; Cons : constructions ; Eng : énergie ; Hyd : hydrologie ; Lim : limites administratives ; Oro : orographie ; Reg : région toponymique ; Veg : végétation.

Fig.4. Le modèle des données vectorielles de la BDTopo50k

\subsection{Le développement des processus de calcul climatologiques}

La particularité de notre solution est concrétisée par le développement des processus de traitement à distance dans le serveur cartographique dédiés aux calculs climatologiques, l'utilisateur aura à sa disposition des outils de calculs hébergés dans le serveur cartographique, ce qui justifie le choix du serveur GeoServer qui prend en charge le service web de géotraitement (Web Processing Service).

\subsubsection{Les outils logiciels}

Après une étude comparative entre les logiciels existants, nous avons opté pour les solutions suivantes :

- GeoServer : est un serveur cartographique open source développé en Java, il respecte les normes de l'Open Geospatial Consortium (OGC) et implémente les services WMS, WFS et les services WCS et WPS en extension (Geoserver, 2016). Il possède une interface 
graphique ce qui implique une facilité d'utilisation, et il se base sur la bibliothèque GéoTools ce qui lui rend compatibilité avec différents types de données (PosteGIS, ShapFile...).

- GeoExt : L'API (Application Programming Interface) GeoExt est une librairie JavaScript qui donne la main à réaliser des interfaces cartographiques riches et personnalisées. Elle est à l'origine d'une fusion entre OpenLayers et d'ExtJS (bibliothèque des applications web interactives) (GeoExt, 2014).

-Apache : c'est le serveur Web/HTTP choisi pour la réalisation de notre solution, il permet la diffusion des données des serveurs cartographiques sur le réseau afin de répondre aux demandes des utilisateurs, via leurs navigateurs avec des pages HTML.

- PostGreSqI/PostGIS : il s'agit d'un système de gestion de base de données relationnelle (SGBD) avec sa cartouche spatiale. Ce système est le plus abouti dans le domaine du logiciel libre. il a été utilisé pour stocker les données géographiques relatives au terrain ainsi que les données climatiques récupérées sous forme tabulaire à savoir: la température, la pluie, l'humidité relative, la pression atmosphérique et la vitesse des vents.

- QGIS : le Quantum GIS (QGIS) est un Système d'Information Géographique libre et Open Source, à travers ses fonctionnalités l'utilisateur peut visualiser, gérer, éditer et analyser ses données (QGIS, 2015), il a été utilisé pour la présentation, la visualisation et la création des styles des données géographiques représentant certains phénomènes climatiques tels que : les orages, les brumes humides, le brouillard, les brumes sèches, les brumes de sable et les tempête de sable. Ainsi, la mise à jour de la BDTopo50k et le géoréférencement et la vectorisation des cartes de l'ANRH et le CRER ont été réalisée par ce logiciel qui offre des outils d'édition avancée.

\subsubsection{Le Web Processing Service}

Le Web Processing Service (WPS) ou le service web de géotraitement est un standard de l'OGC permettant de réaliser en mode web service des traitements sur les données spatiales pour répondre aux besoins de développement de modules de géotraitement par internet, de facilité le partage des algorithmes et des fonctionnalités de géotraitement [21], et ce au profit 
des utilisateurs distants afin de mettre à leurs disposition des outils de géotraitement tels que : le calcul de distance, la bufférisassions,... ou encore des outils de calculs climatologiques comme nous allons le montrer dans la suite de cet article.

\subsubsection{Les requêtes d'un WPS}

Le WPS possède trois (03) requêtes [21]:

- GetCapabilities : cette requête demande au serveur de lister les traitements disponibles à son niveau.

-DescribeProcess : décrit un processus de manière plus détaillée avec ses entrées et sorties.

-Execute : est utilisée pour exécuter les processus spécifiés; c'est avec cette requête que le serveur réalisera en réalité le traitement.

La figure 05 ci-dessous, représente le principe de communication entre un client et un serveur WPS.

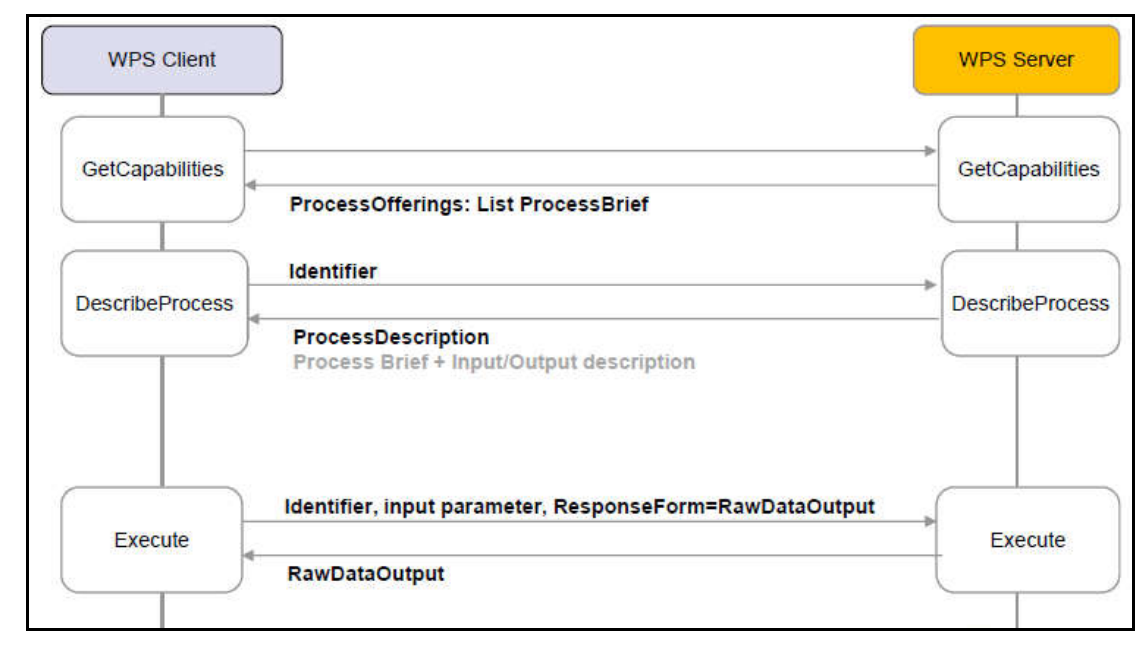

Fig.5. Principe de fonctionnement d'un WPS [23]

\subsubsection{La création de nouveau processus WPS dans notre serveur}

Le module WPS ne fait pas partie du noyau de GeoServer, à cet effet, une installation de l'extension WPS est primordiale. En standard, cette extension propose une liste de processus de géotraitement, sa consultation s'effectuera par l'exécution de la requête GetCapabilities, la réponse du serveur est un document XML appelé le document des capacités (capabilities document) (geoserver, 2016). Néanmoins, les processus dont il dispose ne répondent pas à nos besoins de calculs climatologiques. A cet effet, nous avons procédé à la création des processus 
personnalisés dédiés aux calculs climatologiques. Le développement de ces processus nécessite des connaissances dans la programmation JAVA et XML.

Les processus développés permettront de calculer les facteurs climatiques suivants :

- La température minimale moyenne (T.Min.Moy);

- La température maximale moyenne(T.Max.Moy);

- la variation annuelle de température moyenne mensuelle ( $T$.Annl.Mens);

- la moyenne mensuelle de pluie (P.Mens.Moy);

- L'humidité moyenne (H.Moy);

- La pression atmosphérique moyenne ( $\left.\mathrm{Pa} \cdot{ }_{\mathrm{Moy}}\right)$;

- Le vent moyen (V.Moy);

La figure 6 représente l'interface d'exécution, fournie par le GeoServer, d'un processus de traitement à distance qui permet de calculer la température minimale moyenne entre 1987et 2007.

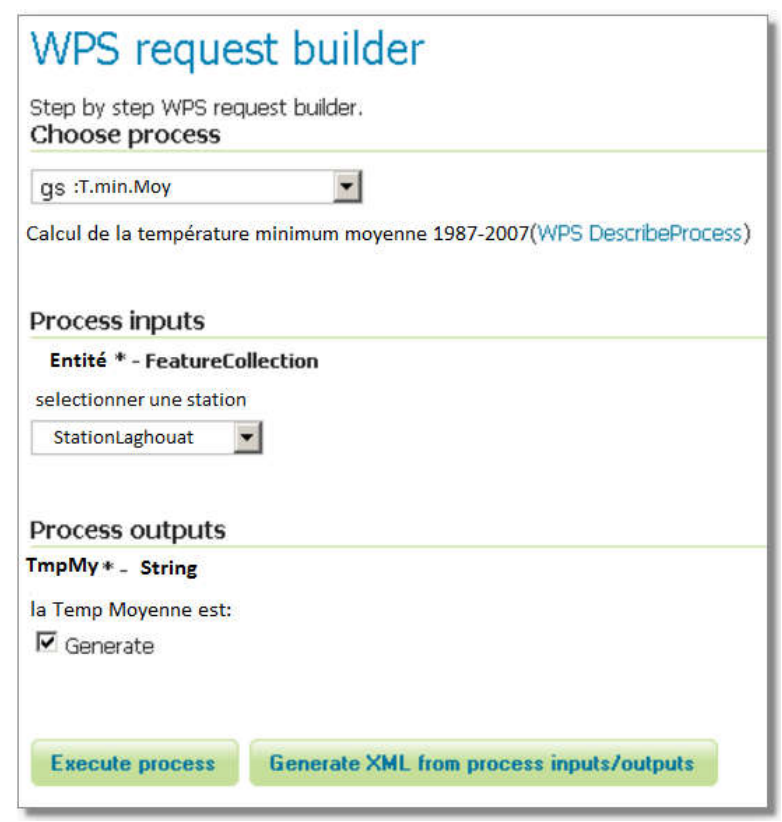

Fig.6. Les paramètres d'entrées et sorties du processus ( $\left.\mathrm{T}_{\text {Min.Moy }}\right)$

Les paramètres d'entrée nécessaires pour l'exécution du processus T.Min.Moy est la couche vectorielle représentant l'emplacement de la station météorologique dans laquelle ont veux calculer la température minimale moyenne sur la période (1987-2007). Après l'exécution, l'utilisateur aura la réponse du serveur sous forme d'un document XML contient des valeurs 
numériques des températures minimales moyennes pour chaque mois (Figure 7).

$<$ ?xml version="1.0" encoding="UTF-8"?>

$<$ ows : TMinMoy>

$<$ ows : jan $>3,0<$ ows : jan $>$

$<$ ows : fev $>4,3<$ ows : fev $>$

$<$ ows : mrs $>6,2<$ ows : mrs $>$

<ows : avl $>9,3<$ ows : avl $>$

$<$ ows : mai $>15,1<$ ows : mai $>$

$<$ ows : jun $>19,7<$ ows : jun $>$

$<$ ows : jui $>23,2<$ lows : jui $>$

$<$ ows : aot $>22,3<$ ows : aot $>$

$<$ ows : spt $>18,4<$ ows : spt $>$

$<$ ows : oct $>12,8</$ ows : oct $>$

$<$ ows : nov $>6,6<$ ows : nov>

$<$ ows : dec $>5,0</$ ows : dec $>$

$<$ ows : TMinMoy $>$

Fig.7. le résultat de l'exécution du processus T.Min.Moy

\section{L'INTERFACE GRAPHIQUE DE LA SOLUTION}

L'interface graphique de la solution développée est représentée sur la figure 9, elle permet l'accès à des outils de recherche, de visualisation. Elle a notamment pour but de publier les données climatiques pour les différents utilisateurs, chacun a un niveau d'accès différent des autres. Dans la même interface l'utilisateur peut aussi consulter des statistiques sur les données climatiques sous forme d'histogrammes et de graphes.

Il est à signaler que dans cette version du système les outils de calculs climatiques ne sont pas accessibles via cette interface, néanmoins, Ils sont exécutables, à partir du WPS Request Builder, une fenêtre intégrée dans l'interface d'administration de GeoServer. 


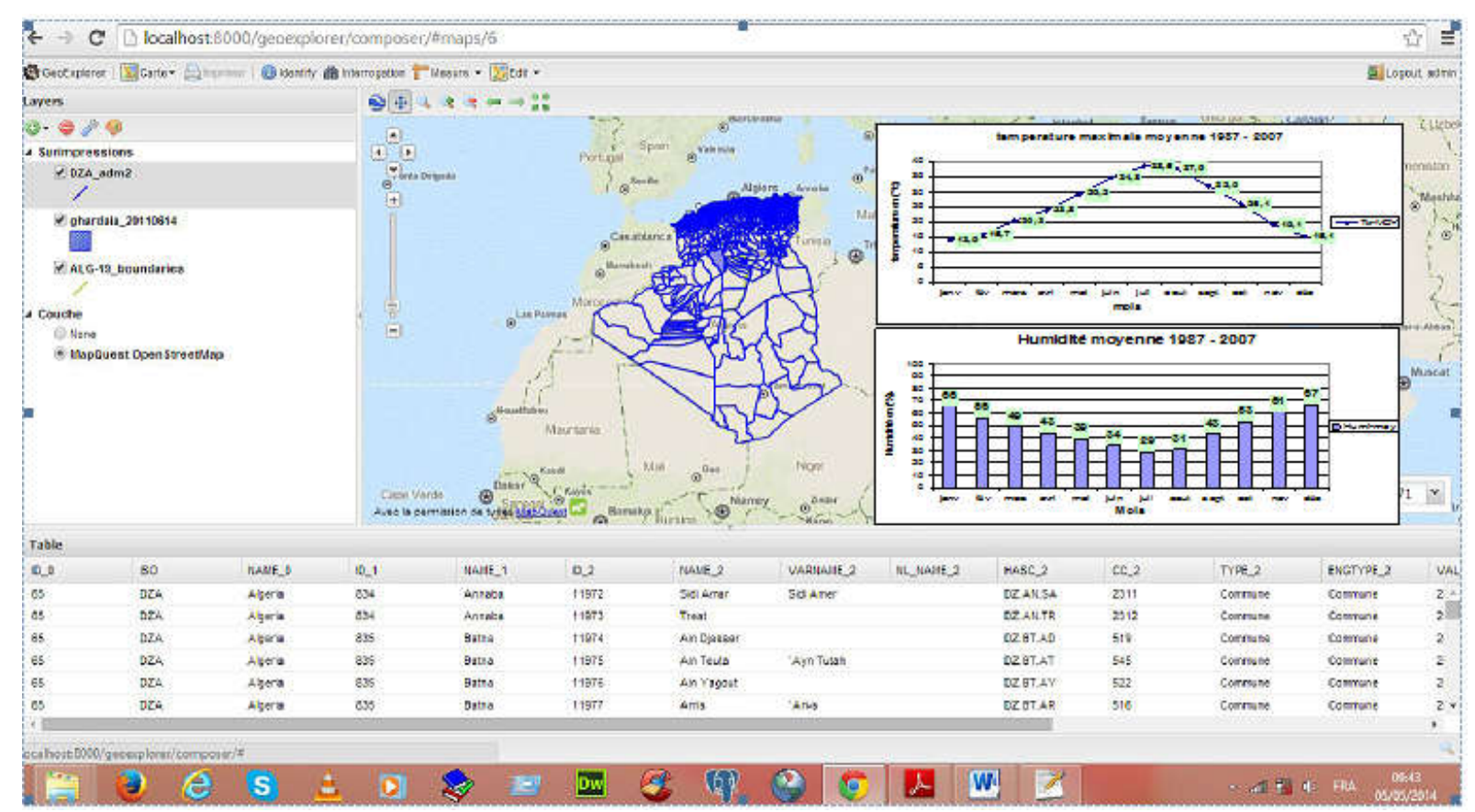

Fig.8. L'interface de la solution SIG-Web pour l'analyse des données climatiques de l'Algérie

\section{CONCLUSION}

Dans cet article nous avons décrit la démarche suivie pour répondre au besoin du Centre Climatologique National qui consiste à mettre en place une solution informatique permettant l'échange des données entre les structures régionales et la structure centrale ainsi que l'exécution des analyses climatiques sur un serveur distant par tous les usagers.

La solution proposée consiste en un Système d'Informations Géographiques sur le Web qui a pour objectif principal l'intégration de données climatiques de l'Algérie dans une base de données centralisée afin de faciliter leur échange d'une façon normalisée. Ainsi, cette solution permet l'exécution des analyses climatiques et l'affichage des résultats sous forme de graphes d'histogrammes.

En plus, notre solution mis à la disposition des utilisateurs des processus dédiés aux calculs climatiques, ils sont développés et hébergés dans le serveur cartographique. Dans la version actuelle l'exécution de ces processus exige l'utilisation de l'interface d'administration du serveur cartographique, ainsi les résultats de ces calculs sont consultables en dehors de l'interface principale de notre solution.

Finalement, la base de données du système ne contient actuellement que les données 
climatiques de quatre stations d'une période de vingt et un ans, néanmoins, elle est ouverte pour l'introduction des données d'une durée plus longue et de toutes les stations météorologiques de l'Algérie, ce qui augmente la capacité d'analyses.

\section{REMERCIEMENT}

Je souhaiterais exprimer ma gratitude à Madame María Teresa Camacho Olmedo; Chef de Département de l'analyse géographique regional et de la géographie physique, de l'université de Granada/Espagne ; pour son aide ainsi que pour ses multiples encouragements.

\section{REFERENCES}

[1] Boasson M, 1995, The Artistry of Software Architecture Maarten, I3E software, vol. 12, No. 6, pp 13-16;

[2] Benlarbi d, d. Boutoutaou, m.1. saker1, 2015, étude de la relation entre le ruissellement, la pluviométrie et l'évaporation des bassins versants de la zone sud de la méditerranée (cas de l'algérie), lebanese science journal, vol. 16, no. 1, pp 3-13 ;

[3] Gardarin G, 2003, Bases de données, Edition 5, Eyrolles, 826 p ;

[4] Gabay J, Gabay D, 2008, UML 2 Analyse et Conception, Mise en œuvre guidée avec études de cas, Dunod, édition 1, 256 pages ;

[5] Joliveau t et al, 2013, La cartographie 2.0, vers une approche critique d'un nouveau régime cartographique. L'information géographique, Armand Colin, 2013, 77 (4), pp 29-46 ;

[6] Reix Robert et al, 2011, Systèmes d'information et management des organisations, édition 6, Vuibert, 480 ;

[7] Pornon H, 2015, SIG: La dimension géographique du système d'information, édition 2, Dunod, $320 \mathrm{p}$;

[8] Pornon $\mathrm{H}$ et al, 2008, Services web géographiques, état de l'art et perspectives, Géomatique Expert, 65, pp 44-50;

[9] Maguire David J, 1991, An overview and definition of GIS, Geographical information systems, volum 2, pp 9-20;

[10] De brichambaut christian perrin, 1993, l'humidité de l'air ; mesures hygrométriques au 
sol, la météorologie, 8e série - $\mathrm{n}^{\circ} 2$, pp 19-26;

[11] DIOT Eric, 2006, La météo de A à Z. Edition Stock, 124 p ;

[12] Nouaceur Zeineddine, 2004, Brume sèche, brume de poussière, chasse-sable et tempête de sable Des types de temps spécifiques des régions sèches, Norois [En ligne], 191 | 2004/2, mis en ligne le 09 septembre 2008, consulté le 16 mai 2016. URL: http://norois.revues.org/1188;

[13] MEBARKI A, 2003, CARTOGRAPHIE AUTOMATIQUE DES PRECIPITATIONS : APPLICATION A L'EST ALGERIEN, Sciences \& Technologie B - N²0, pp. 100-107.

[14] Denègre J et Salge F, 2004, Les systèmes d'information géographique, Paris, Presses Universitaires de France, 127p

[15] Lorho T, 2008, SIGUR:un SIG pour la pratique de l'archéologie en milieu urbain, Archeologia e Calcolatori, pp.61-72;

[16] Deshayes M et al, 2000, SIG, définitions et contraintes de mise en place, Systèmes d'information et informatique et forêt méditerranéenne, $n^{\circ}$ 1, pp 67-69 ;

[17] De man E, 1988, Etablishing a geographical information system in relation to its use : a process of stratigic choice, International journal of geographical information systems, vol 2, pp 245-261;

[18] Jonathan F et Al, 1992, Design models and functionality in GIS, Computers \& Geosciences, Vol 18, N4, pp 387-394;

[19] Laurent J, 2009, Serveur Cartographique et SIG interactifs en ligne, cours SIGMA Toulouse, www.geotests.net ;

[20] ROCHE M, 1963, Hydrologie de surface, GAUTHIER-VILLARS ÉDITEUR, PARIS, $430 \mathrm{p}$;

[21] Bennour T, 2010, le service Web de Géotraitement, mémoire de fin d'études de master, France, $98 \mathrm{p}$;

[22] Laborde J-P, 1991, Méthodes d'interpolation et géostatistiques pour la cartographie automatique à l'usage des géographes, polycopié de cour), Université de Nice, 29 p ;

[23] Arnaud C, 2012, OGC Web Processing Service 1.0 Introduction \& Principes, http://www.rencontres-sig-la-lettre.fr/wp-content/uploads/2012/01/R2012-WPS-Cauchy.pdf 
[24] ANRH, 1993, Carte pluviométrique de l'Algérie du Nord, Ministère de l'Equipement, Projet PNUD/ALG/88/021, Alger, 1 carte et sa notice de 54 p, éd. I.N.C., Alger ;

[25] ANRH, 2002, Carte des évapotranspirations potentielles du Nord de l'Algérie au 1/500 000,). Ministère des Ressources en Eau, 2 feuilles et notice de 42 p, éd. I.N.C.T, Alger ;

[26] ANRH1, 2005, Carte du réseau hydroclimatologique et de la surveillance de la qualité des eaux, Ministère des Ressources en Eau, éd. I.N.C.T, Alger ;

ONM, 2015, www.meteo.dz, le site web de l'office national de météorologie, consulté en Décembre 2015 ;

Meteo France, 2016, www.meteofrance.fr, le site web de l'office national de météorologie, consulté en janvier2016 ;

Geoserver, 2015 :www.gerserver.com, le site web de GeoServer, consulté en décembre 2015 ; GeoExt, 2015, www.GeoExt.org, le site web de GeoExt, consulté en décembre 2015.

QGIS, 2015, www.qgis.org, le site web de Quantum GIS, consulté en décembre 2015.

Apache, 2015, httpd.apache.org, le site web d'apache, consulté en décembre 2015.

ANRH, 2015, www.anrh.dz, le site web de l'Agence Nationale des Ressources Hydrauliques ; CRER, 2015, portail.cder.dz, le site web du Centre de Développement des Energies Renouvelables;

Tomcat, 2015, tomcat.apache.org, le site web d'Apache Tomcat, consulté en décembre 2015 ; INCT, 2015, www.inct.mdn.dz, le site web de 1'Institut National de Cartographie et de Télédétection Algérien, consulté en décembre 2015.

\section{RÉSUMÉ}

En Algérie, la mise en œuvre, le contrôle et l'exploitation des données climatologiques, l'élaboration et la diffusion des publications à caractère périodique ainsi que de l'assistance climatologique aux usagers, sont des tâches du Centre Climatologique National (CCN). Par ailleurs, la gestion efficace de ce volume important de données, nécessite le recours aux nouvelles technologies, tels que les Systèmes d'Information Géographique (SIG). Dans ce contexte, le présent travail consiste à mettre en place une solution informatique sur le WEB, basée sur l'utilisation des Systèmes d'Information Géographique. La démarche suivie consiste, 
en premier lieu, à concevoir des bases de données pour stocker les différentes données utilisées (thématiques et de référence) en prenant en compte les besoins fonctionnels tels que la diffusion de données en plusieurs modes (lecture, téléchargement) et la possibilité d'accès direct aux données depuis des sites éloignés. Ensuite, le choix des technologies et les outils nécessaires pour l'implémentation de la solution cartographique qui permettra, outre les fonctionnalités de stockage et de diffusion des différents types de données, d'analyser des observations climatiques par l'utilisation d'un service web respectant la norme WPS (Web Processig Service).

Mots clés : SIG, SIG-CLIMALG, données climatiques, WPS.

\section{How to cite this article:}

Bennour T, Guettouche MS, Matinez Ibrra E. Sig-climalg: the geographical dimension of climate data in Algeria. J. Fundam. Appl. Sci., 2017, 9(3), 1251-1372. 